\title{
CoV-SRMO - Faible risque pour les Canadiens
}

\author{
Saboui M. ${ }^{1 *}$, Reyes-Domingo F. ${ }^{1}$, Levreault E. ${ }^{1}$, Mersereau T. ${ }^{1}$ \\ ${ }^{1}$ Centre de l'immunisation et des maladies respiratoires infectieuses (CIMRI), Agence de la santé publique du Canada, \\ Ottawa (Ontario). \\ *Auteure-ressource : Myriam.Saboui@phac-aspc.gc.ca
}

\section{Résumé}

Le coronavirus du syndrome respiratoire du Moyen-Orient (CoV-SRMO) est un nouveau coronavirus qui a causé un certain nombre de cas acquis dans la communauté et des éclosions liées aux soins de santé en Arabie saoudite et aux Émirats arabes unis, ainsi que des cas sporadiques dans d'autres pays, particulièrement au Moyen-Orient. Les preuves à ce jour établissent un lien entre les cas d'infection par le CoV-SRMO et l'exposition aux chameaux, y compris aux produits du chameau, ou à des cas probables ou confirmés d'infection par le CoVSRMO chez l'humain. Elle se manifeste généralement sous forme de maladie respiratoire aiguë et est associée à un taux de mortalité de $35 \%$. Selon les renseignements disponibles à ce jour, le risque actuel pour les Canadiens de contracter l'infection par le CoV-SRMO est considéré comme faible. Toutefois, le Comité du Règlement sanitaire international sur le CoV-SRMO a souligné que la recrudescence des cas observés au printemps dernier (2014) peut être un facteur de prédiction d'une augmentation du nombre de cas liés au Hadj, pèlerinage annuel à La Mecque, en Arabie saoudite, qui a eu lieu au début du mois d'octobre 2014. Même si le risque global est faible, l'Agence de la santé publique du Canada et ses Laboratoires nationaux de microbiologie (LNM) en étroite collaboration avec les partenaires provinciaux et territoriaux, le Réseau des laboratoires de santé publique du Canada (RLSPC) et des experts en prévention et en contrôle des infections ont élaboré un certain nombre de documents d'orientation et de protocoles de préparation pour lutter contre le risque d'un cas importé d'infection par le CoV-SRMO au Canada.

\section{Introduction}

Le nouveau coronavirus a été décelé pour la première fois en avril 2012 et a ensuite été appelé le coronavirus du syndrome respiratoire du Moyen-Orient (CoV-SRMO) à sa première apparition dans la Péninsule arabique. Le virus continue de circuler à grande échelle dans cette région géographique. Même si on ne comprend pas entièrement la source, il semble y avoir un lien étroit entre le virus qu'on retrouve chez les chameaux et les personnes touchées. Les coronavirus sont une grande famille de virus qui peuvent causer un éventail de maladies infectieuses, d'un rhume bénin au syndrome respiratoire aigu sévère (SRAS) (1), responsable de l'éclosion grave de 2003 à Toronto, en Ontario et dans un certain nombre d'autres pays. Le CoV-SRMO est génétiquement distinct du virus du SRAS et contrairement au SRAS, le CoV-SRMO ne semble pas se transmettre facilement d'une personne à l'autre (2, 3).

Les principaux symptômes d'infection par le CoV-SRMO comprennent la difficulté à respirer, la fièvre, la toux, la douleur musculaire, la douleur thoracique, les vomissements et la diarrhée. Dans les cas graves, la maladie peut progresser jusqu'à une défaillance polyviscérale entraînant le décès $(1,4)$. On a signalé des cas (la plupart secondaires) qui ont obtenu un résultat positif pour le virus en l'absence de symptômes cliniques (1). À l'heure actuelle, il n'existe pas de traitement antiviral ou de vaccin spécifique disponible pour le CoV-SRMO. Le traitement en est un de soutien et est principalement axé sur les fonctions des organes vitaux (1). Près du tiers des cas se soldent par un décès (5).

Au cours de l'éclosion, il est devenu évident que les travailleurs de la santé dans les pays touchés sont particulièrement à risque d'infection par le CoV-SRMO lorsque des mesures de prévention et de contrôle des infections sous le seuil optimal sont utilisées (1). 
La surveillance continue de l'infection par le CoV-SRMO continue d'être une priorité en matière de santé publique, à la fois au Canada et à l'échelle internationale, tout comme des préoccupations persistent qu'au fil du temps, le CoV-SRMO pourrait se transmettre d'un humain à un autre.

L'année dernière marquait le dixième anniversaire de l'éclosion du SRAS et la plupart des Canadiens sont encore très conscients des répercussions qu'elle a eues sur le Canada. Avec le CoV-SRMO qui en est maintenant à sa deuxième année, les Canadiens continuent de nécessiter et de rechercher des renseignements à propos de son niveau de risque. Depuis le printemps 2013, l'Agence de la santé publique du Canada (l'Agence) a comblé ce besoin de renseignements en réalisant régulièrement des évaluations des risques sur le CoV-SRMO et en publiant des données tant détaillées que sommaires qui comprennent des renseignements épidémiologiques en constante évolution et tous les nouveaux résultats scientifiques.

L'objectif de cette évaluation des risques est de décrire la méthodologie, l'évaluation des risques, l'atténuation des risques et le niveau de risque actuel du CoV-SRMO pour les Canadiens qui habitent au pays.

\section{Méthodologie}

Les renseignements compris dans le résumé de l'évaluation des risques sont fondés sur les données probantes actuellement disponibles sur le CoV-SRMO en date de la publication et ils s'appliquent seulement aux personnes vivant au Canada. Les données probantes ou les meilleurs renseignements disponibles pris en compte dans l'évaluation des risques sont tirés des documents publiés, des renseignements recueillis par l'Agence et d'un certain nombre d'organisations internationales en matière de santé publique, y compris :

- Les évaluations des risques, les résumés de mises à jour et les recommandations provisoires pour les groupes à risque de l'Organisation mondiale de la Santé (OMS) (6).

- Les signalements de cas confirmés ou de décès des pays déclarants par le Règlement sanitaire international (RSI).

- Les évaluations des risques du Centre européen pour la prévention et le contrôle des maladies (ECDC) (7).

- Les avis de biosécurité (8) et les directives provisoires concernant la prévention et le contrôle des infections par le CoV-SRMO dans les milieux de soins de courte durée (9) de l'Agence de la santé publique du Canada.

\section{Résumé de la situation de risque}

\section{Résumé de la situation}

Des cas d'infection par le CoV-SRMO ont été détectés principalement au Moyen-Orient, en particulier dans le Royaume d'Arabie saoudite, et également en Jordanie, au Qatar, aux Émirats arabes unis, au Koweït, au Yémen, à Oman, au Liban et en Iran. Des cas liés à des voyages ont également été signalés par onze pays : d'Europe (au Royaume-Uni, en France, en Italie, en Grèce, en Allemagne et aux Pays-Bas), de l'Afrique du Nord (en Tunisie, en Égypte et en Algérie), de l'Asie du Sud-Est (en Malaisie) et de l'Amérique du Nord (aux ÉtatsUnis) (5). Quelques cas de transmission secondaire ont été signalés dans seulement deux des pays mentionnés précédemment (10). Au Canada, aucun cas n'a été détecté (Figure 1). 
Figure 1 : Répartition géographique mondiale des cas d'infection par le CoV-SRMO, en date du 23 septembre 2014

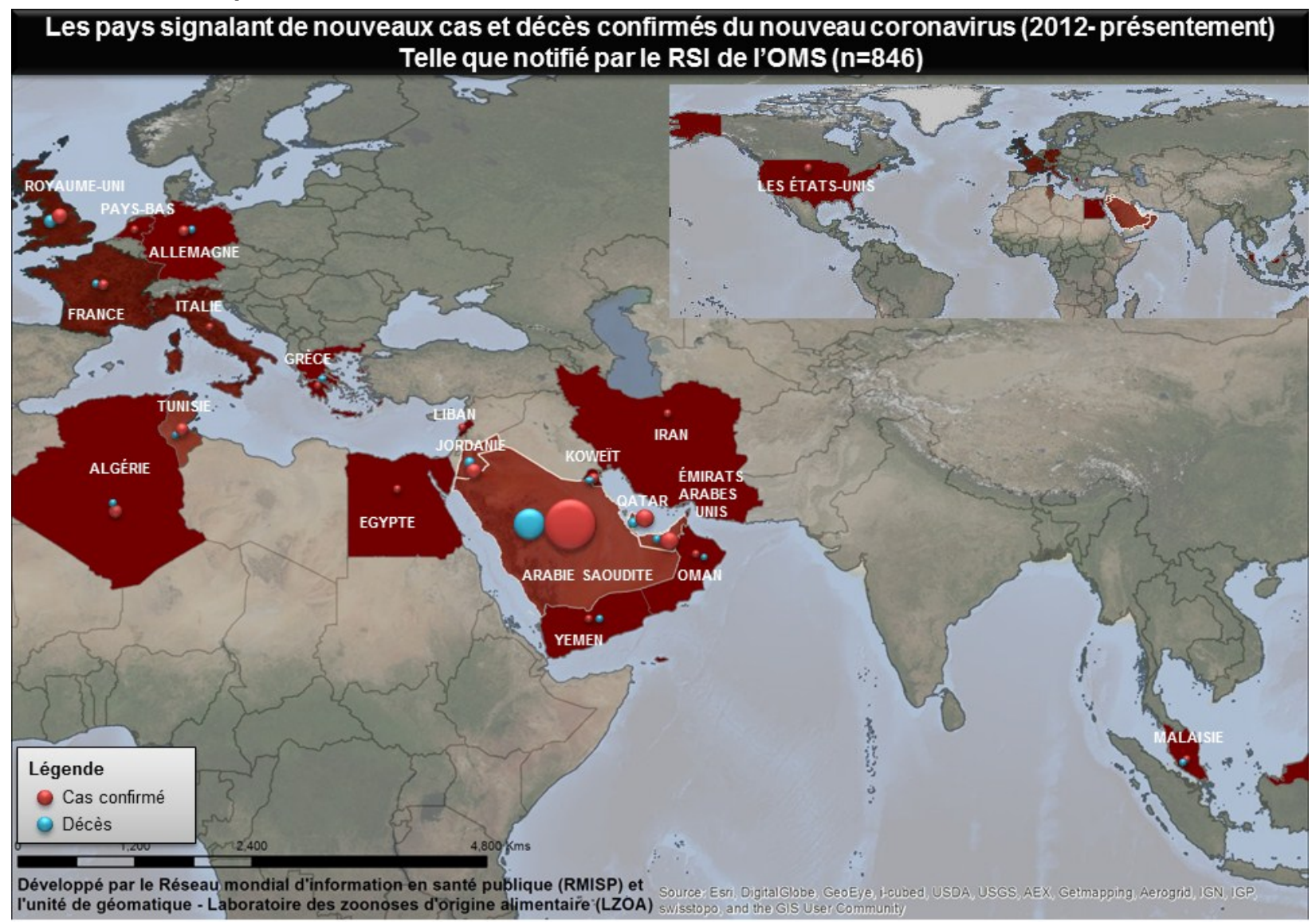

Dernière mise à jour: 23 septembre, 2014, 16:00

+1 Public Heath $\begin{aligned} & \text { Agence de la santé } \\ & \text { Agoncy of Canada } \\ & \text { publique du Canada }\end{aligned}$

Source : Agence de la santé publique du Canada

En date du 23 septembre 2014, l'OMS a signalé 846 cas humains confirmés, dont 298 décès (Tableau 1).

En 2013 et en 2014, on a signalé un nombre accru de cas et de décès dans les mois du printemps. Toutefois, en avril et en mai 2014, une nette recrudescence des cas a été signalée dans le Royaume d'Arabie saoudite, représentant $48 \%$ de tous les cas et $38 \%$ de tous les décès signalés à ce jour (5). Un total de 113 cas rétrospectifs entre mai 2013 et mai 2014 ont été signalés à la fin juin 2014; ils expliquent en partie l'augmentation du nombre de cas signalés au printemps 2014 (11).

Tableau 1 : Cas confirmés d'infection par le CoV-SRMO par pays déclarant en date du 23 septembre $2014 *$

\begin{tabular}{|l|r|r|}
\hline \multicolumn{1}{|c|}{ Pays déclarant } & Cas & \multicolumn{1}{c|}{ Décès } \\
\hline Algérie & 2 & 1 \\
\hline Égypte & 1 & 0 \\
\hline France & 2 & 1 \\
\hline Allemagne & 2 & 1 \\
\hline Grèce & 1 & 1 \\
\hline
\end{tabular}




\begin{tabular}{|l|r|r|}
\hline Iran & 5 & 2 \\
\hline Italie & 1 & 0 \\
\hline Jordanie & 11 & 6 \\
\hline Koweït & 3 & 1 \\
\hline Liban & 1 & 0 \\
\hline Malaisie & 1 & 1 \\
\hline Pays-Bas & 2 & 0 \\
\hline Oman & 2 & 2 \\
\hline Qatar & 7 & 4 \\
\hline Arabie saoudite & 726 & 264 \\
\hline Tunisie & 3 & 1 \\
\hline Émirats arabes unis & 69 & 9 \\
\hline Royaume-Uni & 4 & 3 \\
\hline États-Unis d'Amérique & 2 & 0 \\
\hline Yémen & 1 & 1 \\
\hline Total & 846 & 298 \\
\hline
\end{tabular}

*Source : Communication personnelle - Point de contact régional du RSI de l'OMS, Organisation panaméricaine de la santé et le bureau du point focal national pour le RSI du Canada, septembre 2014.

Selon les données sur les cas disponibles de l'OMS (5), la plupart des cas étaient des hommes adultes (âge médian de mi/fin-quarantaine) présentant des comorbidités et une tendance à être plus gravement malades et à obtenir de moins bons résultats que ceux qui n'en avaient pas. Les personnes atteintes de diabète, d'insuffisance rénale et de maladies pulmonaires chroniques ainsi que les personnes immunodéprimées sont exposées à un plus grand risque de maladie grave si elles contractent une infection par le CoV-SRMO. Les cas secondaires présentent généralement une maladie plus bénigne ou une absence de symptômes; cependant, des maladies graves, dont des décès, ont été observées parmi les cas secondaires, y compris les travailleurs de la santé. La proportion de cas de mortalité est de $35 \%$.

Les données probantes issues des études sérologiques sur les chameaux et du séquençage génétique du virus indiquent que les chameaux sont probablement une source primaire d'infection par le CoV-SRMO chez les humains. D'autres études sur les animaux n'ont pas détecté la présence d'anticorps du CoV-SRMO, y compris celles sur les moutons, les vaches, les chèvres, les buffles d'Inde, les porcs et les oiseaux sauvages (12). Les personnes ayant été en contact avec des chameaux ou des produits du chameau (p. ex. lait cru ou viande, sécrétions ou excrétions (y compris l'urine)) présentaient un risque accru d'infection par le CoV-SRMO. On a également montré que les modes de transmission des cas secondaires dans les milieux de soins et les ménages présentent un risque accru d'infection (5). Le 13 juin 2014, l'OMS a signalé des données probantes à l'appui du rôle des chameaux comme réservoir primaire responsable de l'introduction actuelle du virus dans la population humaine, qui n'entraîne pas une transmission soutenue (12). Seuls quelques cas de transmission au sein des ménages ont été signalés et aucune grosse grappe de cas familiaux n'a été observée (5).

Selon Breben et al. (2013), le taux de reproduction $(R 0)$ du virus s'est révélé inférieur à un $(R 0=0,60)$ et également inférieur au taux de reproduction du SRAS $(R 0=0,80)(13)$. Cependant, les auteurs ont souligné que le taux de reproduction du CoV-SRMO peut augmenter en fonction de la densité de population, et elle peut aussi être influencée par la structure de l'âge et des contacts dans la population. Cauchemez et al. (2013) ont obtenu des estimations similaires du taux de reproduction pour le potentiel épidémique du CoV-SRMO, mais ont déterminé que les valeurs du taux de reproduction augmentent en cas d'absence de mesures de contrôle des 
infections (14). En outre, la transmissibilité peut augmenter au sein des populations présentant des comorbidités et les taux de reproduction peuvent avoir été sous-estimés, si les cas ont reçu un traitement peu après l'apparition de la maladie. Les auteurs ont conclu que même si l'infection par le CoV-SRMO tend lentement à devenir épidémique, les mesures intensives de santé publique envers les cas jumelées à l'amélioration des techniques de diagnostic devraient être suffisantes pour contenir la propagation de la maladie et atténuer les taux de morbidité et de mortalité.

\section{Évaluation du risque}

Selon les meilleurs renseignements disponibles, le risque actuel pour les Canadiens de contracter l'infection par le CoV-SRMO est considéré comme faible. Cette évaluation est fondée sur les observations et les faits suivants :

- On n'a constaté aucune transmission interhumaine soutenue. Le principal mode de transmission est toujours considéré comme étant le contact direct ou indirect avec des chameaux ou des produits du chameau. Le dépistage intensif des contacts avec le CoV-SRMO a révélé de très rares cas de transmission dans les ménages et il n'y a eu aucune augmentation de la taille ou du nombre de grappes de cas observées chez les ménages. La récente augmentation du nombre de cas signalés au cours de l'été 2014 s'explique principalement par des faiblesses dans les mesures de prévention et de contrôle des infections, ou leur absence, dans les établissements de santé en Arabie saoudite et aux Émirats arabes unis.

- Le tableau clinique de l'infection par le CoV-SRMO n'a pas changé.

- Rien n'indique une propagation à l'échelle internationale et les cas récemment exportés n'ont pas entraîné de retransmission soutenue du virus. Le dernier cas exporté dans un pays à l'extérieur du Moyen-Orient a été signalé à la fin mai 2014 en Algérie. II n'a entraîné aucune propagation secondaire symptomatique ou asymptomatique (15).

- Le potentiel pandémique du CoV-SRMO n'a pas été atteint, et on s'attend à ce qu'il demeure faible en raison des mesures de prévention et de contrôle des infections en place.

- L'Agence, en collaboration avec des experts en matière de prévention et de contrôle des infections, a mis au point des lignes directrices fondées sur des données probantes en matière de prévention et de contrôle des infections aux fins d'utilisation dans les milieux de soins canadiens.

- Le Canada possède de solides infrastructures de surveillance de la santé publique et de soins de santé en plus de protocoles de communication établis.

- Lors de sa sixième réunion du 16 juin 2014, le Comité d'urgence du Règlement sanitaire international sur le CoV-SRMO est parvenu à un consensus sur le fait que les conditions d'une urgence de santé publique de portée internationale (USPPI) n'avaient pas encore été réunies (16). La prochaine réunion du Comité est prévue le 25 septembre 2014.

\section{Atténuation du risque}

Dans l'éventualité d'une importation du CoV-SRMO au Canada, l'Agence et le Laboratoire national de microbiologie (LNM), en étroite collaboration avec les partenaires provinciaux et territoriaux (PT), le Réseau des laboratoires de santé publique du Canada (RLSPC) et des experts en prévention et en contrôle des infections ont élaboré un certain nombre de documents d'orientation et de protocoles en matière de préparation (Tableau 2). Depuis 2009, le Canada et ses partenaires provinciaux et territoriaux ont mis en place de solides systèmes de surveillance et de communication conçus pour détecter et signaler les agents pathogènes émergents comme le CoV-SRMO à l'aide du Système de surveillance des infections respiratoires aiguës sévères (IRAS). De plus, le Réseau de surveillance des cas sévères (SOS) du Réseau de recherche sur l'influenza de l'Agence de la santé 
publique du Canada et des Instituts de recherche en santé du Canada (PCIRN), composé de 45 hôpitaux participants destinés aux adultes dans tout le Canada, est conçu pour détecter les admissions en unité de soins intensifs à l'hôpital en raison d'infections respiratoires aiguës sévères.

Tableau 2 : Documents pertinents sur le CoV-SRMO de l'Agence de la santé publique du Canada

\section{Documents pertinents sur le CoV-SRMO de l'Agence de la santé publique du Canada}

1. Résumé de l'évaluation du risque pour la santé publique au Canada associé au CoVSRMO (10)

2. Conseils de santé aux voyageurs pour le CoV-SRMO (17)

3. Avis de biosécurité sur le CoV$\underline{S R M O}(8)$

4. Directives provisoires concernant la prévention et le contrôle des infections par le CoV-SRMO dans les milieux de soins de courte durée (9)

5. Lignes directrices nationales provisoires sur la surveillance des cas d'infection humaine par le CoV-SRMO (18)
Le résumé de l'évaluation du risque est souvent mis à jour et il fournit la plus récente évaluation du risque pour les Canadiens.

Ce document formule des recommandations à l'intention des voyageurs en matière de voyages dans des pays touchés par le CoV-SRMO. Même s'il n'y a eu aucun signalement d'un lien direct entre l'acquisition de l'infection par le CoV-SRMO en raison d'une participation à des rassemblements de masse comme le Hadj et l'Oumra, on conseille toujours aux voyageurs de prendre des précautions avant de se rendre en Arabie saoudite ou dans d'autres pays du Moyen-Orient touchés par le CoV-SRMO.

Cet avis renferme des renseignements qui aident les laboratoires à mettre en œuvre les procédures de biosécurité adéquates pour la manipulation d'échantillons contenant le CoVSRMO.

Ce document fournit des directives en matière de prévention et de contrôle des infections (PCl) à l'intention des organisations de soins de santé et des travailleurs de la santé concernant la gestion des patients qui se présentent dans des établissements de soins de santé au Canada avec une infection suspectée ou confirmée par le CoV-SRMO.

Ce document fournit des lignes directrices en matière de surveillance pour la détection précoce et le confinement des cas d'infection par le CoV-SRMO au Canada. 


\section{Discussion}

Les récents résultats d'enquête sur le CoV-SRMO appuient la théorie selon laquelle les chameaux jouent un rôle important dans l'actuelle transmission du virus. Toutefois, il existe plusieurs cas où une exposition aux chameaux n'a pu être déterminée. Par conséquent, la relation entre l'humain et l'animal dans cet événement exige une étude plus approfondie afin de mieux comprendre les facteurs de risque et les mécanismes sous-jacents associés à la transmission et aussi pour déterminer des mesures efficaces dans la prévention de la plus ample propagation zoonotique (1). Les enquêtes sur les éclosions devront mettre l'accent sur la recherche des contacts pour déterminer les cas peu sévères ou asymptomatiques et pour améliorer les connaissances sur le risque potentiel de transmission ultérieure (5). Ces renseignements sont nécessaires pour déterminer avec précision la meilleure utilisation des ressources de santé publique en cas d'importation d'un cas au Canada.

II existe toujours des préoccupations sur la possibilité de transmission du CoV-SRMO lors de rassemblements de masse comme le Hadj et la Oumra en Arabie saoudite. Toutefois, une étude de séroprévalence menée en 2013 auprès de pèlerins adultes au cours du Hadj de cette année-là n'a pas détecté d'infection par le CoV-SRMO dans l'un ou l'autre des 5235 échantillons testés (19). En outre, on n'a signalé aucun cas confirmé d'infection par le CoV-SRMO associé aux pèlerinages du Hadj de 2012 et de 2013. La recrudescence de cas observée au cours du printemps dernier laissant croire à une augmentation de la circulation de la population peut prédire une augmentation proportionnelle du nombre de cas liés à l'événement Hadj à venir entre le $1^{\mathrm{er}}$ octobre et le 6 octobre 2014 (16).

\section{Conclusions}

Pour l'instant, le risque pour la santé publique au Canada associé au CoV-SRMO est considéré comme faible selon les renseignements disponibles à ce jour. L'Agence, par l'intermédiaire de la surveillance de l'événement en cours, effectue régulièrement et en temps opportun des évaluations du risque posé par le CoV-SRMO par les Canadiens, et elle fournira ces renseignements en temps opportun.

\section{Remerciements}

La contribution des quatre auteurs a été égale. Les auteurs tiennent à remercier le point de contact régional du RSI de I'OMS et le bureau du point focal national pour le RSI du Canada pour avoir fourni le nombre de cas confirmés d'infection par le CoV-SRMO et de décès qui y sont attribuables, par pays.

\section{Conflit d'intérêts}

II n'y a aucun conflit d'intérêts à déclarer.

\section{Références}

(1) Organisation mondiale de la Santé. Foire aux questions sur le coronavirus du syndrome respiratoire du Moyen-Orient (MERS-CoV). Genève : Organisation mondiale de la Santé; 2014.

Accès : http://www.who.int/csr/disease/coronavirus_infections/faq_sep12/fr/.

(2) Organisation mondiale de la Santé. Foire aux questions sur le nouveau coronavirus. Genève : Organisation mondiale de la Santé; 2012. Accès : http://www.who.int/csr/disease/coronavirus_infections/faq_sep12/fr/

(3) Organisation mondiale de la Santé. Foire aux questions sur le nouveau coronavirus. Genève : Organisation mondiale de la Santé; 2012. Accès : http://www.who.int/csr/disease/coronavirus_infections/faq_dec12/fr/

(4) Agence de la santé publique du Canada. Coronavirus du syndrome respiratoire du Moyen-Orient - Symptômes. Ottawa : ASPC; 2014. Accès : http://www.phac-aspc.gc.ca/eri-ire/coronavirus/merscov-symptoms-symptomes-fra.php

(5) Organisation mondiale de la Santé. Middle East respiratory syndrome coronavirus (MERS-CoV) summary and literature update-as of 11 June 2014. Genève: Organisation mondiale de la Santé; 2014.

Accès : http://www.who.int/csr/disease/coronavirus_infections/MERS-CoV_summary_update_20140611.pdf 
(6) Organisation mondiale de la Santé. Alerte et action au niveau mondial (GAR) - Infections à coronavirus. Genève : Organisation mondiale de la Santé; 2014. Accès : http://www.who.int/csr/disease/coronavirus_infections/fr/

(7) Centre européen pour la prévention et le contrôle des maladies. Coronavirus infections - Risk assessments. Stockholm: ECDPC; 2014. Accès : http://ecdc.europa.eu/en/healthtopics/coronavirusinfections/Pages/publications.aspx

(8) Agence de la santé publique Canada. Avis de biosécurité : Coronavirus du syndrome respiratoire du Moyen-Orient (MERS-CoV). Ottawa : ASPC; 2013. Accès : http://www.phac-aspc.gc.ca/lab-bio/res/advi-avis/hce-che-2013-01-22fra.php

(9) Agence de la santé publique du Canada. Directives provisoires - Coronavirus du syndrome respiratoire du MoyenOrient (MERS-CoV) - Lignes directrices sur la prévention et le contrôle des infections dans les établissements de soins actifs. Ottawa : PHAC; 2013. Accès : http://www.phac-aspc.gc.ca/eri-ire/coronavirus/guidance-directives/nCoV-ig-dpfra.php

(10) Agence de la santé publique Canada. Résumé de l'évaluation du risque associé au coronavirus du syndrome respiratoire du Moyen-Orient (MERS-CoV) pour la santé publique au Canada. Ottawa : ASPC; 2014. Accès : http://www.phac-aspc.gc.ca/eri-ire/coronavirus/risk_assessment-evaluation_risque-fra.php

(11) Organisation mondiale de la Santé. Coronavirus du syndrome respiratoire du Moyen-Orient (MERS-CoV) - mise à jour. Genève : Organisation mondiale de la Santé; 2014. Accès : http://www.who.int/csr/don/2014_06_26/fr/

(12) Organisation mondiale de la Santé. Update on MERS-CoV transmission from animals to humans and interim recommendations for at-risk groups. Genève : Organisation mondiale de la Santé; 2014. Accès : http://www.who.int/csr/disease/coronavirus_infections/MERS_CoV_RA_20140613.pdf/

(13) Breban R, Riou J, Fontanet A. Interhuman transmissibility of Middle East respiratory syndrome coronavirus: estimation of pandemic risk. Lancet. 2013 Aug 24; 382(9893):694-699.

(14) Cauchemez S, Fraser C, Van Kerkhove MD, Donnelly CA, Riley S, Rambaut A, et al. Middle East respiratory syndrome coronavirus: quantification of the extent of the epidemic, surveillance biases, and transmissibility. Lancet Infect Dis. 2014 Jan; 14(1):50-56.

(15) Organisation mondiale de la Santé. Middle East respiratory syndrome coronavirus (MERS-CoV) - update. Genève : Organisation mondiale de la Santé; 2014. Accès : http://www.who.int/csr/don/2014_05_22_mers/en/

(16) Organisation mondiale de la Santé. WHO statement on the Sixth Meeting of the IHR Emergency Committee concerning MERS-CoV. Genève : Organisation mondiale de la Santé; 2014.

Accès : http://www.who.int/mediacentre/news/statements/2014/ihr-emergency-committee-merscov/en/

(17) Gouvernement du Canada. Coronavirus du syndrome respiratoire du Moyen-Orient (MERS-CoV) : Conseils de santé aux voyageurs. Ottawa : ASPC; 2014. Accès : http://voyage.gc.ca/voyager/sante-securite/conseils-santevoyageurs/nouveau-coronavirus

(18) Agence de la santé publique du Canada. Lignes directrices nationales provisoires sur la surveillance des cas d'infection par le coronavirus du syndrome respiratoire du Moyen-Orient (MERS-CoV). Ottawa : ASPC; 2013.

Accès : http://www.phac-aspc.gc.ca/eri-ire/coronavirus/guidance-directives/mers-cov-srmo-fra.php

(19) Memish ZA, Assiri A, Almasri M, Alhakeem RF, Turkestani A, Al Rabeeah AA, et al. Prevalence of MERS-CoV nasal carriage and compliance with the Saudi Health recommendations among pilgrims attending the $2013 \mathrm{Hajj}$. J Infect Dis. 2014 Apr 15. 